\title{
A High Precision Electronic Scale Based on STM32
}

\author{
Jiahui Chen \\ Department of Electronic and Communication Engineering, North China Electric Power University, \\ Baoding 071000, China \\ www.cjh161514@qq.com
}

Keywords: Electronic scales, Resistance strain gauge, STM32.

\begin{abstract}
A kind of high precision electronic scale with STM32F103 as the control core is designed. Mainly by the resistance strain gauge, operational amplifiers, ADC and other functional modules. Strain gages of the bridge circuit will be based on the pressure of different output voltage, through the amplifier to get a significant voltage change. Using 24-bit etc. digital-analog conversion chip, through the STM32 acquisition voltage. And the matrix keyboard design input unit price, peeled, calculate the total and overweight alarm and LCD screen display and automatic voltage calibration and other functions.
\end{abstract}

\section{Introduction}

A Electronic scale manufacturing technology and application of ever-changing electronic weighing technology from static weighing to dynamic weighing development: measurement method from analog to digital measurement development; Especially for fast weighing and dynamic weighing of the research and application. But on the whole, China's electronic scale product quality and quality compared with the industrialized countries there is still a gap.

By analyzing the development of electronic scale products this year and the needs of domestic and foreign markets, the development trend of electronic scales must be small, modular, integrated, intelligent; its technical performance trends are high speed, high stability, high stability, The reliability is high; its functional trend is weighing control information and non-control information of both "intelligent" function; its application performance tends to comprehensive and combination. Based on this, this paper presents a small, low-power, intelligent electronic weighing system.

\section{The Principle of High - Precision Electronic Scale}

\subsection{System principle}

The MCU of the high-precision electronic scale is STM32F103 from ST. Mainly by the weighing signal acquisition circuit, keyboard, LCD, communication interface circuit, power management circuit and other parts of the circuit. Its schematic diagram is shown in Fig 1.In Fig.1, the weighing senor; differential amplifier circuit and A/D conversion circuit contribute to the weighing signal acquisition circuit.

When the scale loaded things, the weighing sensor which is mounted on a cantilever beam would produce a voltage signal proportional to the object being tested. After amplification, differential amplifier filtering, A/D transition, and then the signal would be sent to the MCU. Complete the weighing signal acquisition. And the final measurement values are obtained through the software filtering algorithm in MCU, and displayed in the LCD. System used the keyboard to achieve data input electronic scales, accumulate, and peel and other functions.

Electronic scale has RS232 interface can communicate with the host computer function, the system has a normal operating mode and power saving mode, the power management circuit can achieve low power consumption design. 


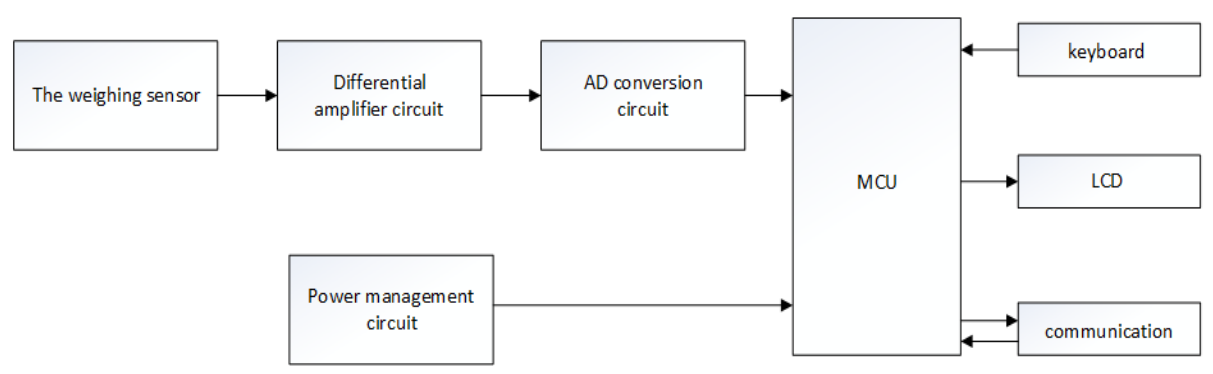

Fig.2 Huygens Bridge

\subsection{The work principle of the weighing sensor}

The performance of the weighing sensor has a lot influence on the accuracy of electronic scale. The electronic scale adopts resistance strain gauge to convert mass into electrical signal. Resistance strain-gauge transducer selects four resistances strain gauge to paste on the elastic sensitive material. Forming the four resistance strain piece Huygens Bridge [4] .When the elastic sensitive material doesn't get under pressure. The resistance strain gauge is not deformed. Therefore, the output voltage of the Huygens Bridge is 0.Conversely, when the elastic sensitive material get under pressure, bridge lost balance and output voltage signal which is directly proportional to the mass of the object. The Resistance strain-gauge transducer's principle is shown in Fig.2. In Fig.2, set four resistances are R1, R2, R3 and R4.The Huygens Bridge's supply voltage is, so the output voltage U1 is

$$
U 1=\left(\frac{R 4}{R 3+R 4}-\frac{R 2}{R 1+R 2}\right) U .
$$

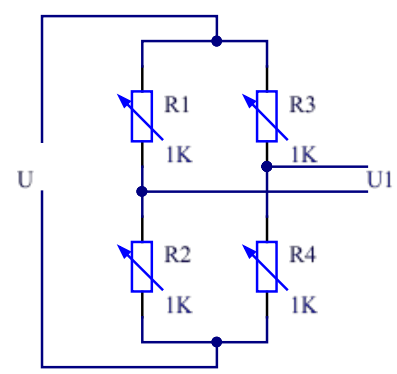

Fig.2 Huygens Bridge

The value of resistance is equal. In this case:

$$
\left\{\begin{array}{l}
R 1=R 2=R 3=R 4 \\
\Delta R 1=\Delta R 3=\Delta R \\
\Delta R 2=\Delta R 4=\mu \Delta R
\end{array}\right.
$$

In (2), is the Poisson's ratio of elastic material. is the variation of resistance. When the sensor is subjected to an object.R1 and R3 are extended. The valve of R1 and R3 has increase.R2 and R4 are compressed. The valve of R1 and R3 has reduced. Bring the second formula into the first formula. Get a new formula.

$$
U 1=\frac{1+\mu}{8}\left(\frac{\Delta R 1}{R 1}+\frac{\Delta R 2}{R 2}+\frac{\Delta R 3}{R 3}+\frac{\Delta R 4}{R 4}\right)
$$

Formula (3) shows that the output voltage is proportional to the change of the value of the resistance strain gauge. 


\section{The Hardware Design}

\subsection{Circuit index calculation}

Using the instrumentation amplifier LT1782, its low offset voltage, low noise and high bandwidth, ideal for small signal processing. As the maximum load current of the amplifier is $4 \mathrm{~mA}$, so set the input voltage of $2.5 \mathrm{v}$, the resistance of $1 \mathrm{~K}$, made the output of $2.5 \mathrm{~mA}$ constant current source, the strain gauge resistance of 120 ohms, take Huygens Bridge And gain amplification through the differential amplifier. A / D converter reference voltage is $3.3 \mathrm{v}$, to leave a certain margin, we use 10 times the magnification. And set its operating frequency of $20 \mathrm{HZ}$, to ensure its sampling accuracy of 21 bits.

\subsection{Power supply part of the circuit}

Power part of the chip power consumption is small, with a good load capacity and temperature characteristics, stable output $\pm 3.3 \mathrm{~V}$. The right side of Fig. 3 is a charge pump voltage inverter, which converts the positive voltage to the corresponding negative voltage.

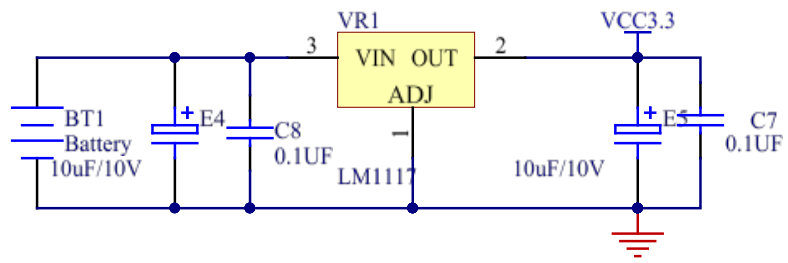

(a)

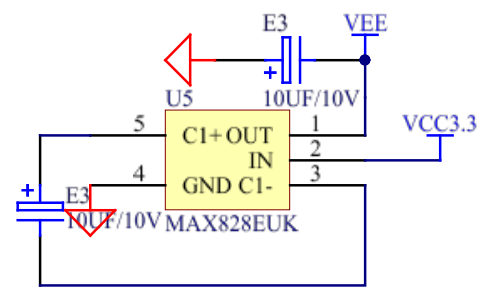

(b)

Fig.3 power supply schematic circuit

\subsection{Constant current source circuit}

Constant current source circuit shown in Fig.4, according to the op amp short features, so that the load current stability at $3.3 \mathrm{~mA}$, measured by the current stability is good.

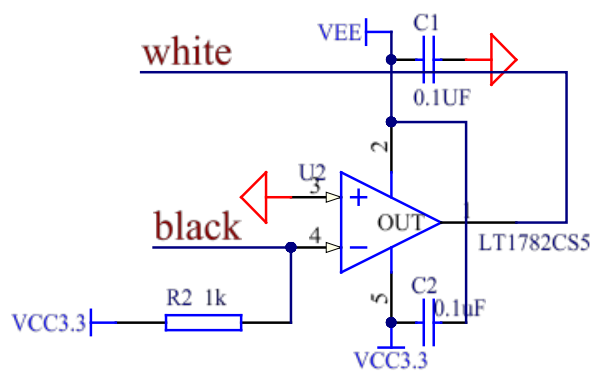

Fig.4 constant current source supply schematic circuit

\subsection{Gain amplification and A / D acquisition part of the circuit diagram}

Gain amplification and A / D acquisition circuit schematic diagram shown in Fig.5. 


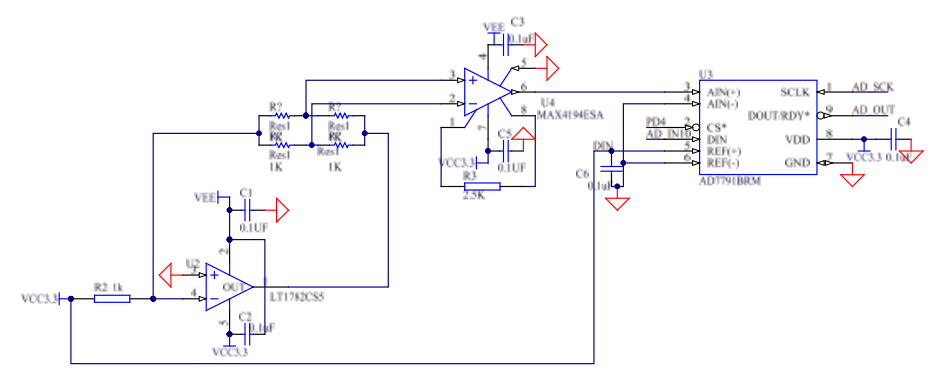

Fig. 5 AD acquisition part of the schematic

The amplifier is selected for the MAX4194 with rail-to-rail voltage output, zero temperature drift, and minimal offset voltage. According to the following formula can be calculated magnification.

$$
V_{\text {out }}=\left(\text { Vin }_{+}-\text {Vin }_{-}\right) \cdot\left[(2 \cdot R 1) / R_{G}\right]+1
$$

$\mathrm{AD}$ part of the reference voltage acquisition circuit and the current source to provide the same voltage, so when the REF changes, the current source to provide the current will change, which makes the AD circuit has good stability.

\section{The Software Design}

\subsection{Signal acquisition and conversion}

1)AD Acquisition Filtering Algorithm:AD collected data and can not be excluded due to external interference caused by the short jump data acquisition, so the method of averaging 5 times only some of the hopping data (before and after the difference is too large) to delete, does not affect the actual collection of AD Accuracy.

2)The algorithm between the $\mathrm{AD}$ acquisition value and the actual conversion: As a measure of the use of iron material, and can not be restored after each deformation, can not return to the initial size, but we found that the quality of each increment after the change is certain, after several measurements, we Using the incremental multiplied by the average slope as the conversion formula.

\section{2 function fitting}

The sampled values of AD and actual mass are fitted into a curve in MATLAB (Fig. 6). Roughly in line with the linear curve, the fitting of more than $96 \%$. In order to improve the processing speed of SCM and better fitting, we use the segmentation function, in 0-400g and 400-500g choose different slope calculation and evaluation.

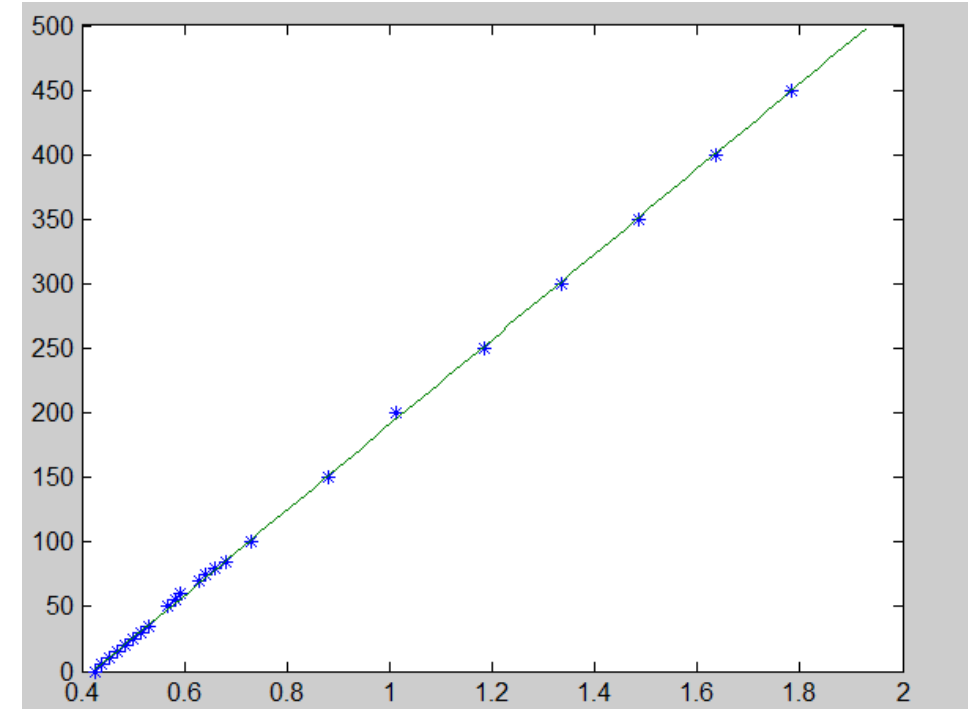

Fig.6 AD sample values and the relationship between the weight of items 


\section{The System Test}

Weighing weights of different sizes (5mg, 1g, 5g, 10g, 20g, 50g, 100g, 500g) put in different scales (electronic weighing scale and precision electronic scale) weighing less than $100 \mathrm{~g}$ When the error is less than $0.5 \mathrm{~g}$, greater than $100 \mathrm{~g}$ when the error within $1 \mathrm{~g}$.

The test results are shown in the TABLE1:

\begin{tabular}{|c|l|l|l|l|}
\hline $\begin{array}{c}\text { Actual } \\
\text { quality }\end{array}$ & \multicolumn{1}{|c|}{$5 \mathrm{~g}$} & $10 \mathrm{~g}$ & \multicolumn{1}{|c|}{$50 \mathrm{~g}$} & $100 \mathrm{~g}$ \\
\hline $\begin{array}{c}\text { Test } \\
\text { quality }\end{array}$ & $4.9 \mathrm{~g}$ & $10.1 \mathrm{~g}$ & $49.8 \mathrm{~g}$ & $100.3 \mathrm{~g}$ \\
\hline $\begin{array}{c}\text { Actual } \\
\text { quality }\end{array}$ & $200 \mathrm{~g}$ & $300 \mathrm{~g}$ & $400 \mathrm{~g}$ & $500 \mathrm{~g}$ \\
\hline $\begin{array}{c}\text { Test } \\
\text { quality }\end{array}$ & $200.3 \mathrm{~g}$ & $299.7 \mathrm{~g}$ & $400.4 \mathrm{~g}$ & $499.5 \mathrm{~g}$ \\
\hline
\end{tabular}

\section{The Conclusion}

The weighing system to achieve the load within $100 \mathrm{~g}$ error within the scope of 0.5 grams, more than $100 \mathrm{~g}$ error in less than 1 gram. Using low power chips, can be powered directly from the battery, using 24-bit ultra-high precision AD7791, to identify changes in smile, and AD reference voltage and op amp input voltage for the same voltage, when the voltage changes in real time Calibration, enhance the circuit stability and practicality, because the strain gauges used in the system deformation is too large, limiting the range at $500 \mathrm{~g}$, using the appropriate deformation strain gauge, you can expand the range.

\section{References}

[1] A. Ajovalasit,L. D'Acquisto,S. Fragapane,B. Zuccarello. Stiffness and Reinforcement Effect of Electrical Resistance Strain Gauges[J]. Strain,2007,434:.

[2] Dian-Ji, Lv,Jian-Sheng, Peng,Guo-Juan, Zhou,Yong, Xu. A High Precision Multi-Function Electronic Scale Based on PSoC3[J]. Sensors \&amp; Transducers,2013,192:..

[3] Steven P. Reiss. Automated and Algorithmic Debugging[B]. Lecture Notes in Computer Science, 2005..

[4] Burch C B. Huygens' pulse models as a bridge between phenomena and Huygens' mechanical foundations.[J]. Janus; revue internationale de l'histoire des sciences, de la médecine, de la pharmacie, et de la technique,1981,68:.. 